TIPA. Travaux interdisciplinaires sur la parole et le langage

$34 \mid 2018$

La langue des signes, c'est comme ça

\title{
Langue des signes et parentalité : enjeux linguistiques et identitaires
}

Sign language and parenthood: linguistic and identity issues

Sarah Kirsch et Charles Gaucher

\section{OpenEdition}

1 Journals

Édition électronique

URL : http://journals.openedition.org/tipa/2605

DOI : $10.4000 /$ tipa.2605

ISSN : 2264-7082

Éditeur

Laboratoire Parole et Langage

Référence électronique

Sarah Kirsch et Charles Gaucher, «Langue des signes et parentalité : enjeux linguistiques et identitaires », TIPA. Travaux interdisciplinaires sur la parole et le langage [En ligne], 34 | 2018, mis en ligne le 12 septembre 2018, consulté le 10 décembre 2020. URL : http://journals.openedition.org/tipa/2605 ; DOI : https://doi.org/10.4000/tipa.2605

Ce document a été généré automatiquement le 10 décembre 2020.

La revue TIPA. Travaux interdisciplinaires sur la parole et le langage est mise à disposition selon les termes de la licence Creative Commons Attribution - Pas d'Utilisation Commerciale - Pas de Modification 4.0 International. 


\title{
Langue des signes et parentalité : enjeux linguistiques et identitaires
}

\author{
Sign language and parenthood: linguistic and identity issues
}

\author{
Sarah Kirsch et Charles Gaucher
}

Ce média ne peut être affiché ici. Veuillez vous reporter à l'édition en ligne http:// journals.openedition.org/tipa/2605

\section{Introduction}

2 La naissance d'un enfant présentant une surdité déstabilise de manière générale le projet familial initialement prévu par les parents. La surdité marque l'environnement des familles (White \& Klein, 2008); lorsque les parents apprennent le diagnostic, ils passent par différentes émotions, dont l'inquiétude de ne pouvoir communiquer avec leur enfant. Ils vont questionner leur rôle de parent, alors qu'il reste fondamentalement le même (Gaucher \& Duchesne, 2015), mettant en cause ce que certains appellent le parentage intuitif ${ }^{1}$. Ces nouveaux parents entendants d'enfant sourd sont rapidement mis en contact avec de nombreux services (audiologiste, orthophoniste, oto-rhino-laryngologiste, etc.) et sont amenés à s'engager activement dans tout le processus de soutien social de leur enfant. Au Canada, les familles sont censées recevoir un diagnostic très tôt dans la vie de leur enfant et profitent par la suite d'un accompagnement de professionnels avant l'âge de six mois. Pourtant, l'accès à des services adaptés à leur réalité et en français représente un défi pour des familles. Effectivement, pour certaines provinces, les services spécialisés sont proposés uniquement dans des régions anglodominantes et par des institutions qui peinent souvent à leur offrir un réel accompagnement dans leur langue maternelle. Les spécificités des provinces influencent sur la prestation et la diversité des services proposés aux familles francophones d'enfant ayant une surdité. La répartition 
géographique de la population ne facilite pas l'accès aux différents choix de services pour ces familles.

3 À cela s'ajoute la nécessité de faire différents choix pour l'enfant sourd: le langage parlé complété (LPC), l'oralisation, les langues signées, l'implantation, l'appareillage, etc. C'est avec l'accompagnement des professionnels de la santé, de la réadaptation et du milieu de l'éducation que les parents devront s'impliquer dans le cheminement qui leur semble le plus adéquat pour leur enfant sourd.

De tous ces choix, ceux relatifs au mode de communication adopté entre le parent et l'enfant déterminent leur expérience de parentage. Alors que l'implant cochléaire est devenu une procédure presque automatique dans les cas de surdités profonde et sévère au Canada ${ }^{2}$, il semble dorénavant évident que seulement une minorité de parents choisiront les langues signées dans leur trajectoire communicationnelle avec leur enfant sourd. Les parents peuvent penser que la puissance du nouvel outil qu'est l'implant et que l'hégémonie avec laquelle le monde médical applique cet outil annule ou atténue le besoin de recourir à une langue signée pour communiquer avec l'enfant sourd. Le présent article vise à questionner « cette évidence » afin de voir, du point de vue des parents, quels sont les obstacles qui peuvent entraver, dans leur expérience de parentage, leur désir d'apprendre et de transmettre une langue signée à leur enfant sourd. Nous posons l'hypothèse que les parents entendants s'engagent dans les mesures d'accompagnement offert à leur enfant sourd en ayant pour souci de minimiser les sentiments d'incompétence, d'incompréhension et d'incertitude produits par la remise en cause du parentage intuitif qui accompagne l'annonce de la surdité de leur enfant. Nous tenterons de voir comment, à la lumière de témoignages recueillis dans le cadre d'une recherche menée auprès de parents canadiens, l'option "langue des signes " constitue souvent pour eux une source de malaise qui peut être compris comme un frein à leur engagement.

5 L'article s'intéressera d'abord à la place de la surdité dans l'élaboration des mesures de soutien au Canada. Quelques précisions méthodologiques seront ensuite présentées afin de mettre en contexte les données et les analyses discutées dans l'article, lesquelles proviennent d'une étude portant sur l'expérience des parents venant de trois pays: Canada, France et Belgique. Nous nous concentrerons par la suite sur l'impact que l'annonce de la surdité peut avoir sur la trajectoire familiale ainsi que sur l'engagement parental. Dans la continuité de cette réflexion, nous verrons que l'idée d'inclure une langue signée au sein de l'environnement familial peut engendrer une gamme de sentiments en rapport à la transmission linguistique et à la transmission identitaire qui posent problèmes aux parents quand vient le temps de réfléchir à la question de l'apprentissage d'une langue signée comme façon de communiquer avec leur enfant.

\section{La surdité comme lieu d'intervention social}

6 Depuis les années 1970, de multiples changements ont eu cours dans les services offerts aux personnes handicapées au Canada (Boucher, 2001 ; Fougeyrollas, 2010), en France (Stiker, 1982) et en Belgique (Vrancken \& Bartholomé, 2004). Ces transformations ont d'autant plus influé sur le domaine de la surdité qui était, encore à cette période, marqué par l'institutionnalisation des enfants présentant une déficience auditive (pour le Québec: Perreault, 1996, 2002 et 2006; Lachance 2007; Gaucher, 2009 - Pour la France : Delaporte, 2002). Le changement qui s'est opéré a été marqué non seulement 
par la façon de considérer le handicap dans notre société, mais aussi par les nouvelles méthodes et philosophies d'intervention dites «intégrationnistes " qui ont profondément bouleversé la façon de considérer la surdité (Lachance, 2002 et 2007), et ce, surtout auprès des enfants d'âge scolaire (Lane, 1993; Mottez, 1977 ou, pour une perspective radicalement critique, Ladd, 1983). Pour les parents vivant avec un enfant ayant des incapacités auditives, il n'était plus seulement question de se demander s'ils allaient ou non confier leur enfant à une institution spécialisée ; tout un univers de choix et de positionnements s'est ouvert à eux quant à l'accompagnement de leur enfant vers une inclusion optimale. Les clivages éducatifs dans les philosophies d'enseignement (Delaporte, 2002, Cuxac, 1983 pour la France ; Lachance, 2007 pour le Québec ou encore Lane, 1984 pour les États-Unis) vont fortement marquer le ton de ces années de transition et la question de la langue enseignée aux enfants deviendra un enjeu sensible qui, tout en évoluant, marquera définitivement les modes d'inclusion des enfants vivant avec une surdité (Gaucher, 2007) et donc nécessairement le rôle de leurs parents. Des études québécoises abordent le vécu de quelques parents à partir d'histoires de vie (Dubuisson \& Grimard, 2006) et des recherches françaises interrogent l'incidence de la surdité sur la fratrie (Bouteyre et al., 2006), sur la communication dans la famille (Mottez, 2006) ou les dynamiques entre parents et jeunes sourds (Lagarrigue, 2011). Ces études n'abordent que de façon générale ou périphérique le triangle relationnel parents-enfant-acteurs impliqués dans les services visant l'inclusion sociale. Toutefois, les rapports entre les intervenants et les parents d'enfant ayant des incapacités en général peuvent être quelquefois tendus à cause du décalage qu'ils ressentent entre leurs attentes mutuelles (Dionne et al., 2006). Nous connaissons par contre peu les impacts de ces tensions sur la façon dont les parents s'engagent dans les mesures de soutien visant l'inclusion des enfants handicapés, ce qui est d'autant plus vrai pour les enfants vivant avec une surdité. Selon différentes études anglo-saxonnes, la surdité affecte les parents, voire la famille entière et transforme les rapports parentaux. Des études américaines ont montré que l'interface entre les professionnels qui s'occupent d'enfants ayant des incapacités auditives et leurs mères est un espace sensible qui peut devenir un agent stressant pour celles-ci (Calderon \& Greenberg, 1999). Nous savons également de façon plus particulière que des tensions sont produites par les différents dilemmes que rencontrent les parents lorsqu'ils doivent faire des choix concernant les services proposés à leur enfant (Young et al., 2005 et Luterman, 1987). Si ces dilemmes deviennent trop envahissants, certains parents ne se laissent toutefois pas submerger par ces tensions avec les instances responsables de les éclairer dans leur choix; plusieurs vont chercher de l'information où ils peuvent, en tentant de comprendre ce qui arrive à leur enfant (Porter \& Edirippulige, 2007). Encore faut-il que ces informations soient disponibles, pertinentes et intelligibles pour eux, ce qui, malheureusement pour les parents francophones du Canada, n'est pas toujours le cas.

\section{Méthodologie}

7 Le point de départ de notre recherche est la question de l'engagement des parents francophones dans les services proposés à leur enfant vivant avec une surdité. Il s'agit d'une recherche internationale et comparative effectuée auprès de 100 parents francophones vivant au Canada, en Belgique, en France et en Suisse. Notre article s'appuie sur l'échantillon canadien de la recherche comprenant 52 entrevues semi- 
dirigées menées de 2014 à 2015 auprès de parents francophones d'enfant ayant une surdité légère, moyenne, sévère ou profonde vivant au Québec ou au NouveauBrunswick. Un appel à participation a été fait par l'Association québécoise des enfants avec problèmes auditifs (AQEPA) pour le Canada. Les parents étaient invités à contacter les chercheurs s'ils souhaitaient participer à la recherche. Sur 52 participants rencontrés (36 mères et 16 pères), seulement 3 avaient choisi la langue des signes québécoise (LSQ) comme principale langue de communication pour leur enfant sourd et 6 l'avaient utilisée à un moment ou à un autre. Ainsi, cet article explore les représentations des parents au sujet des langues signées qui pourraient aider à comprendre, au-delà de certaines idées reçues qui circulent dans le monde des Sourds et qui supposent que les institutions médicales «manipulent » les parents, pourquoi si peu de familles font le choix d'une langue signée pour leur enfant ayant une surdité ${ }^{3}$.

Ces entrevues qualitatives d'une durée moyenne de 60 minutes visaient d'abord, dans une perspective ethnographique, à documenter l'expérience des parents en général. Elles ont permis de recueillir des témoignages imprégnés de la question des choix communicationnels. Les entretiens du corpus de données ont été transcrits et analysés selon les principes de l'analyse de contenu (Deslauriers, 1997; Bardin, 1986). En lien avec les objectifs poursuivis et le canevas d'entrevue, l'expérience générale et la participation des parents aux services proposés à leur enfant sourd étaient les deux catégories d'analyses initiales. Ce travail de catégorisation a été réalisé à l'aide du logiciel Nud*ist NVivo (Qualitative Research Solution, Pty Ltd, Australia). Tout le matériel ainsi divisé par catégories a été relu afin d'identifier les noyaux de sens qui constituaient l'essentiel du phénomène étudié. Nous avons porté une attention particulière aux éléments qui allaient à l'encontre de la tendance principale, l'implication des parents comme quelque chose allant de soi, afin d'apporter les nuances aux multiples espaces d'engagement. Cette façon de procéder a permis d'éviter de prendre appui sur un seul point de vue en tentant de comprendre la diversité des formes d'engagement des parents et des rapports qu'ils entretiennent avec les acteurs qui sont impliqués auprès de leurs enfants. Les entrevues ont été nommées sous un principe de codage, les chiffres suivant les extraits permettent, entre autres, de retrouver les informations concernant les participants. Par exemple, 12-505-1, le nombre 12 nous permet d'identifier la province, le 505 nous indique le numéro d'identification de l'entrevue, et le 1 chiffre le sexe de l'interviewé. La question de la langue des signes et des modes de communication a été abordée sous plusieurs angles dans les entrevues et a été répertoriée dans diverses catégories du cahier de codage, catégories qui nous ont servi à produire les analyses présentées dans cet article. Les trois principales catégories utilisées pour formuler notre hypothèse de départ concernant le rapport entre les parents, les acteurs impliqués dans le domaine de la surdité et les divers modes de communication sont les suivantes: "contact avec communauté sourde et langue des signes", "appréciation des modes de communication et des technologies (appareillage, implant, langue des signes, etc.) » et "perception des modes de communication ».

9 C'est à partir de ce traitement des données recueillies que nous avons tenté de produire quelques pistes d'interprétation sur les obstacles linguistiques et identitaires liés au choix d'une langue signée comme principal mode de communication. 


\section{L'engagement aujourd'hui}

10 La question de l'engagement, qui accompagne et accélère le démantèlement de l'État providence, souligne, comme le disaient Charbonneau \& Estèbe il y a de cela de plus de dix ans, la multiplication des «appels en faveur d'un renouvellement du contrat social et de la participation de tous à la création d'une nouvelle cohérence sociale" (Charbonneau \& Estèbe, $2001: 6$ ). Cet appel à la participation n'est pas que politique : elle inclut toutes les sphères d'action étatiques, voire plus profondément de nature collective. Ainsi, l'engagement comme mode de mise en relation des individus implique une redéfinition du rapport entre liens contractuels et liens obligés. En reprenant les acceptions conventionnelles du concept d'engagement, Charbonneau \& Estèbe écrivent, en parlant de la responsabilité du citoyen contemporain, qu'il s'agit pour les nouveaux acteurs sociaux de « le convaincre, le faire accepter volontairement de s'engager envers les autres et de participer à la vie de la cité » (2001: 5). L'engagement est, comme l'écrit Giraud (2011), une promesse. La promesse de s'investir par-delà le nécessaire, de ne pas s'en tenir à ce qui est obligé et de faire, dans un monde de contraintes, une différence auprès d'autrui. S'engager, c'est faire la promesse à un individu, à un groupe, à une cause ou à un idéal que nous sommes disponibles pour plus que ce qu'on attend de nous, que l'on est ouvert à créer de l'espoir. Le souci de faire s'engager les personnes concernées par une situation particulière, par exemple l'inclusion d'un enfant ayant des incapacités auditives, souligne d'une part le fait que la notion d'engagement met en tension l'autonomie et la responsabilité dans la formulation des solutions collectives aux problèmes sociaux et, d'autre part, que cette notion semble dorénavant s'appliquer à la description de liens sociaux qui appartenaient, jusqu'à maintenant à d'autres sphères sociales centrées sur l'espace privé, l'intimité et la proximité (Saillant \& Gagnon, 2001). On est ici devant une extension du contractuel qui semble avoir définitivement réussi à coloniser le monde des relations ayant jusque-là été conçues comme allant d'elles-mêmes (par exemple la relation parents-enfant). En ce qui concerne plus spécifiquement les parents d'enfant ayant des incapacités auditives, la surdité étant « invisible » tant que la communication ne représente pas un enjeu, il est raisonnable de présumer que ceux-ci peuvent avoir de la difficulté à se représenter la singularité de leur enfant, à la concevoir et donc bien souvent à s'engager de façon précoce dans les services qui leur sont proposés. Les divers professionnels impliqués dans les mesures d'inclusion des enfants vivant avec une surdité, de la naissance à leur entrée à l'école, doivent créer un espace où l'engagement puisse naître. Cet espace, souvent marqué par des tensions concernant les savoirs et les savoir-faire, est un lieu qui ne va pas de soi et où l'on exige souvent beaucoup des parents (Gardou, 1996), particulièrement dans le domaine de la surdité, puisque les interventions visant l'inclusion mettent en jeu un médium de socialisation central au développement même de l'enfant, soit le langage et la communication. Or, peu d'études dans le domaine de la surdité ont jusqu'à présent tenté de cerner cet espace d'engagement où se négocient l'aide et le soutien entre des acteurs qui ont non seulement des intérêts différents, mais qui sont de nature différente: parents, grands-parents, fratrie, intervenants communautaires, professionnels des services étatiques, etc. L'espace d'engagement qui s'offre aux parents est multiforme et complexe, non seulement à comprendre, mais à s'approprier. 


\section{Présentation des données}

\section{Surdité, engagement et choix parentaux}

11 Lorsque le diagnostic de la surdité est annoncé, les parents peuvent recevoir cette information comme un choc, les précipitant subitement dans un monde qui leur est étranger (Jackson et al., 2004). Hintermair (2000) a fait ressortir dans son étude effectuée en Allemagne que 9,5\% des parents ont eu des contacts avec des adultes sourds avant la naissance de leur enfant sourd. Une grande partie des parents se sentent désorientés dans ce nouveau monde qu'est la surdité (Flaherty, 2015). Sans repères face à cette annonce, si ce n'est les perceptions, les stéréotypes et les préjugés communs, ces parents se trouvent devant une série de prises de décisions, dont la première touche le mode de communication de leur enfant. Les familles où naît un enfant sourd, généralement entendantes, vont avoir tendance à s'engager dans une voie qui répond à leur identité et à ce qu'elles connaissent: la langue orale comme unique mode de communication (Dalle-Nazébi, 2014).

12 Après avoir annoncé la surdité de l'enfant, les professionnels jouent un rôle prépondérant dans l'accompagnement des parents ainsi que dans la présentation des possibilités qui s'offrent à l'enfant sourd et ses parents. Cependant, dans le cas d'une surdité profonde et sévère de l'enfant, l'implant est généralement la solution unique proposée (Lhéricel, 2006). Certains intervenants ne présentent pas les alternatives, ce qui participe de manière déterminante à la perception des familles à propos des différents modes de communications pour l'enfant vivant avec une surdité (Dubuisson \& Grimard, 2006). Plusieurs parents ont exprimé n'avoir eu qu'un choix limité concernant les options communicationnelles.

Je n'ai pas choisi la voie oraliste, c'est le centre de réadaptation qui a considéré que pour lui, c'était le côté oraliste. (12-301-3)

Assurément, si j'avais su ou si on m'avait dit qu'avec son implant, il n'entendra pas tout... Il faudrait garder le langage des signes, parce que dans certaines situations (...) on aurait pu se parler en signes et ça aurait été correct. (12-103-1)

Les professionnels ne m'ont jamais parlé d'utiliser un langage codé comme le LPC, le français signé ou encore la langue des signes. Probablement que l'audiologiste voyait qu'il en n'avait pas besoin. Ils ne m'ont jamais parlé de ça. (12-514-1)

Dans plusieurs cas, les parents n'ont pas l'impression d'avoir choisi l'implant; les professionnels l'ont présenté comme la réponse la plus logique à la surdité. Pour les parents qui « veulent toujours le mieux pour leur enfant ", l'implant devient l'option qui s'impose. Cette situation ne renforce pas le sentiment de compétence du parent n'ayant pas l'impression d'avoir pris part à la décision.

Les langues signées deviennent ainsi pour plusieurs parents des "plans B». Si les $a$ priori négatifs vis-à-vis de ces langues de certains professionnels semblent avoir un rôle à jouer dans cette attitude, ils n'expliquent cependant pas tout. Nous partons du point de vue que les parents ne sont pas simplement des individus influencés par les institutions sociosanitaires et que leurs choix sont aussi motivés par des sentiments et expériences qui renvoient à des processus anthropologiques profonds. La prochaine 
section vise justement à aborder le discours propre aux parents afin de leur laisser la parole pour comprendre, de leur point de vue, ce qui peut entraver leur choix de se diriger vers une langue signée pour communiquer avec leur enfant et l'éduquer.

\section{Transmettre une langue qui n'est pas la sienne}

En ce qui concerne le choix de s'orienter ou non vers une langue signée, des parents interrogés ont émis différentes explications, en lien avec la transmission linguistique, pour expliquer leur décision. Ces explications, souvent en porte-à-faux les unes avec les autres, sont des interprétations subjectives qui ressortent des entretiens réalisés et analysés. D'une part, elles renvoient à l'idée que les langues signées sont "simples » et qu'il est « inutile » de les enseigner, elles « viendront d'elles-mêmes » à l'enfant sourd. D'autre part, les parents ont le sentiment que les langues signées sont des obstacles à l'inclusion de leur enfant et donc, les leur transmettre serait aller contre le « gros bon sens ».

- Les langues signées viennent «naturellement » aux personnes sourdes :

Pour certains parents interrogés, les langues signées sont intrinsèques aux personnes sourdes et, si elles peuvent être envisagées dans les premiers temps suivant la naissance de l'enfant, il demeure inutile de se préoccuper de leur transmission :

On avait commencé avec mon mari au début, puis ce n'est pas très compliqué, donc si elle le fait régulièrement ça va se faire tout seul. (12-401-1)

17 Si une langue signée est envisageable par les parents dès la naissance de l'enfant, c'est souvent, ainsi que le rappelle Millet (1999), à titre de mesure de soutien pédagogique pour amener l'enfant vers une langue orale. Toutefois, pour beaucoup de parents rencontrés dans le cadre de notre recherche, les langues signées doivent venir après coup, une fois que l'enfant a acquis la langue de ses parents. Millet (2003) dit même que les langues des signes peuvent devenir pour les parents une «langue pour plus tard»:

Un jour elle va peut-être décider de devenir juste LSQ et d'enlever ses implants. C'est elle qui va décider. (12-505-1)

Mais on veut qu'elle se concentre d'abord sur la parole et bien acquérir ce qui est parole, langage, etc. pour ensuite quand elle sera plus grande elle pourra apprendre la langue des signes. (12-401-1)

On s'est dit que s'il voulait aller se plonger dans la culture sourde un peu plus tard il pourra le faire. (12-502-1)

Moi je laisse libre, elle pourra faire son choix. Je ne l'obligerai pas à y aller, si elle veut y aller elle y va. Si elle ne veut pas, c'est son choix aussi. (12-109-2)

Cet apprentissage a posteriori serait possible car, pour plusieurs parents rencontrés, les langues signées sont simples et faciles à apprendre. À travers ces extraits, on sent bien que pour plusieurs parents entendants, les langues signées ne sont pas un objet de préoccupation pour eux; ce sont des langues qui s'acquièrent sans effort. 
On veut qu'elle se concentre sur la parole et bien acquérir ce qui est parole, langage, etc. pour ensuite, quand elle sera plus grande, elle pourra apprendre la langue des signes (...), ce n'est pas très compliqué donc si elle le fait régulièrement, ça va se faire tout seul. C'est plus dur d'apprendre à parler que d'apprendre à signer. (12-401-1)

Malheureusement on a abandonné les signes parce que c'était plus facile que la parole. (12-103-1)

Je me disais voyons, s'il est capable d'apprendre la langue des signes qu'ils ne viennent pas me dire qu'il n'est pas capable d'apprendre à parler. C'est beaucoup plus complexe que d'apprendre des signes. (12-302-1)

Ces familles n'excluent pas définitivement les langues signées, mais l'intérêt de choisir une langue qu'elles perçoivent comme moins complexe n'est pas logique à leurs yeux. Le prestige d'une langue construit par certains parents joue un rôle déterminant dans le choix des langues opéré par les familles (Delamote-Legrand \& Sabria, 2001). La perception que les langues signées sont « simples » joue un rôle prépondérant à notre avis dans la décision des parents de les transmettre à leur enfant sourd.

- Les langues signées isolent les enfants :

Selon plusieurs parents, langue des signes est synonyme d'exclusion sociale. Pour nombre d'entre eux, l'acquisition de la parole, par tous les moyens possibles, demeure garante d'une intégration réussie pour l'enfant au sein de sa famille et aussi, plus généralement, au sein de la société. C'est la voie qui semble aller de soi, celle qui rapproche l'enfant de son milieu «naturel ». En choisissant la parole pour leur enfant, les parents tentent de l'inscrire dans une trajectoire familiale "normale " qui les obligera à entrer dans une logique de réparation de l'enfant (Virole, 2006). Dans les extraits ci-dessous, différentes raisons sont données pour expliquer l'isolement imaginé par les parents qu'aurait pu créer une orientation vers une langue signée.

On a abandonné les signes parce que c'était plus facile la parole. C'est plus normal, donc on veut le plus normal. (12-103-1)

Le langage des signes versus une enfance plus ou moins normale avec de l'audition, on a opté pour l'audition. (11-201-2)

21 D'une part, dans leur discours, ces parents entendants expriment leur inquiétude de socialiser leur enfant à l'aide d'une langue signée dès lors que cette langue risque de renforcer l'isolement du jeune sourd. Plusieurs facteurs sont évoqués pouvant expliquer l'inquiétude de ces parents de voir leur enfant ayant une surdité, isolé de par son mode de communication:

Quand on nous présente la surdité, on pense tout de suite : 'oh mon Dieu! Langage des signes, isolement. (12-401-1)

Le monde ne signe pas en général, c'est ça la réalité fait que pourquoi le pousser dans un premier temps dans les signes si le monde ne signe pas. (12-101-1) 
D'autre part, quelques parents s'inquiètent de l'usage des signes au sein même de la famille, puisqu'ils craignent que la langue des signes utilisée par l'enfant ne soit pas partagée par tous les membres de la famille. Le choix de l'oralisme est donc fait par les parents pour préserver « la cohésion familiale » (Lhéricel, 2006, citée par Dubuisson \& Grimard, 2006).

Cette enfant-là, elle n'avait pas de communication avec les parents de mon conjoint et une communication so $\mathrm{so}^{4}$ avec son père parce que son père ne connaît pas les signes. Quand même qu'il aurait voulu l'apprendre il n'aurait probablement pas été si fluide que je ne l'aurais été. (12-118-1)

Un autre facteur abordé, est la situation géographique des familles. En effet, dans certaines régions du Canada, les possibilités de s'exprimer en langue signée sont très limitées :

Si j'avais choisi de pas appareiller mon enfant au [région éloignée] il aurait été la culture sourde à lui tout seul... l'implant est un outil qui permet que mon enfant il peut entrer en contact avec qui il veut. Donc pour moi de ne pas lui offrir ça, c'est l'isoler donc je ne peux pas accepter ça. (12-502-1)

Ce sentiment d'isolement peut être augmenté par le manque de contacts réguliers entre parents entendants et adultes sourds. L'inexistence de modèles dans leur environnement influence indubitablement le fait de ne pas apprendre la LSQ et encore plus leur désir de la transmettre.

Les parents ont une représentation réductrice de la nature des langues signées et de leur potentiel. Les témoignages recueillis laissent entrevoir que les parents n'ont pas reçu les « outils» leur permettant de s'engager dans la voie des signes. Apprendre une langue signée pour la transmettre à son enfant devient dans cette mesure un effort « inutile» (elle viendra naturellement de toute façon) et "contreproductif » (elle nuira à l'inclusion sociale de l'enfant). Pour les parents rencontrés, nul doute que d'opter pour une langue signée est un problème fondamental. Ils ont une représentation réductrice de la nature des langues signées et de leur potentiel. Selon eux, il y a une incompatibilité dans la complémentarité de l'apprentissage d'une langue signée et du développement de la parole.

\section{Transmettre la langue d'un Autre}

L'avènement de la surdité implique que le parent prenne un rôle qu'il n'avait pas prévu de jouer. Ce rôle exige une réévaluation de plusieurs pans de sa vie et, par le fait même, une transition identitaire importante. Il est ainsi facile d'imaginer que d'inclure une langue signée dans le portrait familial peut sembler, pour certains parents, une lourde tâche. Considérer une langue signée comme un moyen de communication avec son enfant implique que le parent se sente outillé et informé quant aux possibilités qu'offrent les langues signées. Ces enjeux reliés à la transmission linguistique sont accompagnés d'autres préoccupations de nature identitaire. Tout d'abord, les parents se préoccupent de la pérennité de leur choix éducationnel s'ils vont vers une langue signée. De plus, plusieurs ont avoué se sentir loin des locuteurs de la LSQ et ne pas se 
sentir à l'aise au sein de la communauté sourde qui leur paraît déconnectée de leur réalité.

- Les langues signées sont vouées à disparaître:

Pour plusieurs parents, les langues signées sont en « voie d'extinction ». Étant donné le poids que représente le choix d'un mode de communication déterminant l'avenir de l'enfant, il semble irréaliste pour des parents de choisir une langue dont l'utilisation sera, d'après eux, de plus en plus rare.

Avec les implants, le LSQ va être une langue morte d'ici un siècle. Je ne lis pas dans l'avenir, mais les implants sont de plus en plus populaires. Le pourcentage de gens non opérables est tellement minime, que ça va faire comme le latin. (12-505-2)

La culture sourde telle qu'elle est en ce moment est vouée à disparaître parce qu'on a des outils tellement performants. (12-502-1)

Mais c'est ça, la langue tantôt il n'y en aura plus, puis ça va être rare ceux qui vont avoir la langue des signes. (12-106-1)

Les parents ne voient ainsi pas l'intérêt d'enseigner une langue signée à leur enfant considérant que les langues des signes sont en train de disparaître. Choisir une langue signée constitue pour certains une source d'inquiétudes inutiles. Ce choix représenterait, en quelque sorte, une impasse identitaire.

- L'accès aux locuteurs d'une langue signée est difficile:

Pour nombre de parents entendants, l'intérêt d'enseigner une langue signée à leur enfant est moindre, car dans leur esprit, elles sont génératrices d'un mouvement de fermeture au monde commun. Les enfants ayant une surdité seront à même de pouvoir la maîtriser et s'affilier à la communauté sourde, mais, pour les parents, cela semble plus difficile, voire impossible, de faire partie du monde des Sourds. Il serait donc illogique pour eux d'éduquer leur enfant dans une langue qu'ils ne pourront apprendre que superficiellement et, qui plus est, mènera leur enfant à s'affilier à une communauté qui n'est pas la leur.

Première des choses elle ne parle pas le langage des sourds donc oublie ça, elle ne serait pas la bienvenue dans cette communauté-là. Et moi ce que je perçois là-dedans, c'est que tu es le bienvenu ou tu n'es pas le bienvenu. La communauté sourde c'est une communauté familiale tu sais c'est ... et les étrangers qui rentrent doivent savoir parler sinon ils ne sont pas les bienvenus. C'est comme ça que je le perçois. Oui ils sont bien contents tu as un enfant qui est sourd ils vont te parler mais ça ne va pas durer bien longtemps, ils vont te rejeter du revers de la main et prendre quelqu'un qu'ils savent avec qui ils vont parler.

La culture sourde, ils sont très fermés, on n'est pas invités nous les entendants. Tu les vois dans une cabane à sucre toute la gagne puis ils nous ignorent totalement. Ce n'est pas des gens qui vont faire, veux-tu l'apprendre? Ils ne viennent pas nous chercher.

Nous faisons partie de la communauté sourde, mais c'est la mentalité de fermeture de la communauté sourde gestuelle que j'aime moins. C'est peut-être une fausse idée que j'ai aussi... 
Le sentiment d'éloignement qu'expriment ces parents au regard de la communauté sourde renforce l'idée qu'ils ne sont pas des agents de socialisation compétents en langue signée. De plus, l'idée d'inclure une langue signée dans la trajectoire de la famille augmente les incertitudes du parent quant à sa capacité à inscrire son enfant « dans la continuité des générations » (Dagenais, 2000) dont il est le représentant. Ainsi, la charge identitaire reliée au choix d'une langue signée comme mode de socialisation constitue, à notre avis, un frein pour les parents qui, de par le bouleversement existentiel que produit la surdité sur leur trajectoire, peinent déjà à recomposer leur projet familial.

\section{Discussion}

\section{Choisir une langue signée : source de malaise pour les parents entendants d'enfant sourd?}

31 Les familles qui apprennent la surdité de leur enfant doivent rapidement prendre des décisions concernant leur mode de communication. À la lumière des pistes de réflexion qu'offrent les données recueillies auprès des 52 parents canadiens ayant participé à la recherche, il est raisonnable de croire que durant cette période de transition, les familles qui désirent s'engager dans les mesures de soutien offertes à leur enfant sourd sont confrontées à trois sentiments négatifs qui peuvent définitivement freiner leur élan vers une langue signée comme mode de communication et de socialisation de leur enfant sourd : sentiment d'incompétence, sentiment d'incompréhension et sentiment d'inquiétude (voir tableau 1). Les lignes qui suivent reprennent chacun de ces sentiments pour en faire des propositions provisoires concernant les enjeux linguistiques et identitaires qui nous semblent faire obstacle à l'adoption d'une langue signée par les parents entendants pour la communication et l'éducation de leur enfant sourd au Canada.

- Le sentiment d'incompétence

Les familles ont une responsabilité relative à la vie future de l'enfant ayant une déficience auditive; les décisions qui seront prises en lien avec le choix de la langue déterminent son parcours de vie. Pour Minet (2017), le rôle qu'a le parent d'enfant avec handicap s'appuie sur :

Une double facette de la responsabilité, le pouvoir de prendre des décisions et être garant de ceux dont on a la charge, qui se pose sur le parent, scellant son engagement dans un entremêlement dont il ne pourra s'en sortir (p. 86).

Les répercussions que peuvent avoir le choix éducationnel reposent sur les décisions parentales. Que les parents décident de s'orienter vers une « langue orale » ou vers une langue signée, l'apprentissage de cette langue par l'enfant sourd se fera surtout dans les interactions avec les membres de sa famille (Lhéricel, 2006). Par conséquent, si les parents décident de s'orienter vers une langue signée, cela nécessitera pour ces parents entendants d'apprendre rapidement cette langue pour la transmettre à leur enfant. Cette option nécessite un investissement important de la part du parent qui, dans cette situation, se trouve paradoxalement à apprendre la langue de son enfant. Cette inversion n'est pas sans poser de problèmes, car certains ne considèrent pas avoir les 
compétences nécessaires pour y parvenir. D'autre part, comme l'expliquent Dubuisson \& Berthiaume (2005), en plus de l'apprentissage d'une langue des signes, il ne faut pas oublier le défi qu'est son utilisation dans la vie quotidienne.

Être parent d'enfant sourd demande également des compétences spécifiques associées à l'enfant ayant une déficience auditive (Jackson et al., 2004). Ces compétences nécessitent une gestion du temps de la part des parents à travers le grand nombre de rendez-vous proposés pour le suivi du jeune sourd, mais également dans le processus de prise de décision qui doit se faire dès les premiers mois. D'autre part, un ajustement dans leur communication au sein de la famille et dans les relations sociales est nécessaire pour répondre au mieux aux besoins de l'enfant

Pour ce faire, les professionnels sont amenés à accompagner les parents dans leur cheminement et les aider à se construire en tant que parents. Selon Scelles (2002, cité dans Minet, 2017), il faut que :

La rencontre entre les familles et les professionnels, loin de générer une disqualification mutuelle, conduise chacun des partenaires à bénéficier des compétences de l'autre et ne fasse pas obstacle au déploiement des capacités créatrices de chacun (p. 89).

Pourtant certains parents ne se considèrent pas aptes à prendre certaines décisions et plusieurs parents rencontrés semblent croire, à l'instar de ce que d'autres auteurs ont remarqué, que les intervenants sont ceux qui vont « détenir le savoir » concernant leur enfant (Dubuisson \& Grimard, 2006). D'autres familles, en revanche, n'ont pas le souvenir d'avoir réellement choisi le mode de communication qu'elles utilisent avec leur enfant. Or, si cette collaboration entre parents et professionnels ne permet pas de bénéficier d'un savoir-faire alors on peut s'interroger sur la représentation que ces familles peuvent avoir concernant leurs compétences parentales.

Ainsi, tant sur le plan linguistique qu'identitaire, un sentiment d'incompétence chez certains parents peut émerger lorsqu'ils envisagent d'apprendre une langue qui n'est pas la leur pour la transmettre à leur enfant qui deviendra un locuteur plus habile qu'eux Entre le sentiment d'incompétence et le sentiment d'incompréhension, nous voyons qu'il y a une variation dans la représentation des langues signées pour certaines familles, "cela s'apprend facilement » alors que pour d'autres " cela semble difficile». Les parents sont dans un contexte où le potentiel de ces langues leur est peu ou pas expliqué par des intervenants qu'ils croient plus à même de savoir ce qui est le mieux pour leur enfant. Pourtant, pour Hinermair (2000, citée dans Dubuisson \& Grimard, 2006), les parents qui signent auront de meilleures interactions familiales et ce choix peut aider les parents à mieux accepter la surdité de leur enfant.

- Le sentiment d'incompréhension :

Le deuxième sentiment que nous aimerions aborder est celui d'incompréhension que nous ont semblé éprouver certains parents lorsqu'ils envisagent une langue signée comme mode de communication avec leur enfant. Plusieurs possibilités sont offertes quant aux choix de mode de communication pour un enfant sourd. Pourtant, Millet (2006) met en évidence que, dans les faits, les parents se voient restreints à choisir entre deux possibilités qui reviennent à s'orienter soit vers l'oral soit vers le gestuel. Si l'engagement se fait dans une langue signée, cela implique que les membres de la famille trouvent une alternative à la parole et s'initient aux signes (Dalle-Nazebi, 2014). L'apprentissage de la langue des signes se fait souvent selon une initiative personnelle 
et sans vraiment de soutien des principaux acteurs du milieu de la surdité. Ce choix éducatif transgresse une norme collective relative aux processus de transmission linguistique (Therrien \& Le Gall, 2012) et il engage une réorganisation familiale. Un tel investissement linguistique peut paraître incompréhensible pour certaines familles, surtout si elles pensent que les langues des signes viennent naturellement aux personnes sourdes. En effet, dans les lieux communs concernant la surdité, les langues des signes sont souvent perçues comme intrinsèques aux Sourds (Gaucher, 2012). Cette perception n'épargne bien sûr pas les parents qui sont presque toujours des nouveaux venus dans le monde de la surdité. Elle laisse croire que ces langues sont faciles à apprendre et que le statut de Sourd permet d'échanger presque instinctivement en langue des signes. Il semble ainsi évident pour les parents d'orienter leur enfant vers une langue parlée, cela n'excluant pas que, plus tard, leur enfant ira de lui-même vers une communication gestuelle.

Rappelons que les langues signées ne sont pas transmises selon les mêmes modalités que la plupart des langues orales; elles sont incarnées et apprises par et pour les Sourds (Dalle-Nazebi, 2014). Le frein à l'usage d'une langue signée peut donc venir du fait que le parent entendant craint de briser le lien et d'amener l'enfant à se désaffilier de son réseau familial pour rejoindre celui de la communauté sourde. Cette perception va à l'encontre d'une idée à laquelle les parents sont attachés : l'intégration pour leur enfant (Dubuisson \& Grimard, 2006). De plus, Minet (2017) rappelle que la représentation d'une impossible séparation de la personne handicapée de ses parents scelle la responsabilité parentale (p. 162). Cette séparation est représentée comme difficile pour les parents, étant donné les liens qui les unissent à leur enfant. Par conséquent, il semble incompréhensible pour eux de s'orienter vers une langue «barrière " qui les sépare de leur enfant et qui, de toute façon, fait partie «naturellement » du bagage linguistique que l'enfant sourd pourra choisir plus tard s'il le désire.

- Le sentiment d'inquiétude :

40 Le poids des représentations et des stéréotypes peut influencer la vision qu'ont les parents d'enfant sourd vis-à-vis des langues signées. D'après Delamotte-Legrand \& Sabria (2001),

La vision que les gens se sont ainsi construite des langues hiérarchise selon les moments et les enjeux des critères de sympathie ou d'empathie pour une langue et ses locuteurs, de prestige d'une langue lié souvent à l'idée que l'on se fait de sa richesse culturelle, de la facilité de son apprentissage, de son utilité sociale dans la vie de tous les jours comme dans le devenir professionnel des personnes. (p. 95).

Les familles sont précocement prises en charge par le milieu médical et paramédical, et souvent selon des modalités où les décisions concernant le choix éducatif pour leur enfant sourd doivent être prises rapidement. Les parents ont conscience d'être les premiers éducateurs et modèles pour leurs enfants (Cloutier et al., 2005), mais les conditions dans lesquelles ils doivent prendre des décisions peuvent renforcer chez eux leurs inquiétudes par rapport aux langues signées. Le fait que les professionnels ne présentent pas ce mode de communication comme une véritable option accentue l'idée qu'elles ne sont pas utiles pour leur enfant sourd (Lhéricel, 2006) au point d'être vues comme un vecteur d'exclusion extra et intra familial. De plus, ne connaissant pas de locuteurs utilisant une langue signée, les parents peuvent être sceptiques quant à leur 
«prestige potentiel » considérant qu'elles sont maîtrisées par très peu de locuteurs. Ainsi, pensant que cette langue est toujours minoritaire et se sentant exclus de cette minorité, l'argument le plus récurrent pour ces parents est qu'ils ne souhaitent pas isoler leur enfant au sein de la société (Nader-Grosbois, 2015).

Les langues signées ont longtemps souffert de représentations dépréciatives. Cela explique pourquoi il est encore difficile d'envisager pour ces familles ce mode de communication comme une option valable. La réalité de ce constat est que les familles croient que ces langues sont en train de « disparaître " à cause d'une systématisation des implants cochléaires (Virole, 2011). La dyade entre parent entendant et enfant sourd est décrite en termes de rupture linguistique, culturelle et affective allant de pair avec l'appauvrissement, les difficultés et le dysfonctionnement de la communication (Lavigne, 2003 : 94). L'adoption d'une trajectoire parentale qui placerait l'enfant dans un monde qui s'éteint augmente les angoisses liées à la déficience de leur progéniture et entrave par conséquent leur relation avec leur enfant. Ces parents craignent d'isoler leur enfant au sein de la société, mais également au sein de leur propre famille en utilisant une langue qui pourrait disparaître et que personne ne connaît.

À la lumière des données recueillies et de nos analyses, il semble que ces trois sentiments négatifs alimentent le malaise qui accompagne la remise en question du parentage intuitif vécu par les parents ayant un enfant sourd. Peuvent-ils être abordés de front par les acteurs soucieux de la pérennité des langues signées? Par-delà les discours qui dénoncent la mainmise des institutions sociosanitaires sur les parents, il faut aussi prendre en compte les craintes et les résistances des parents pour ce qu'ils sont : des agents de transmission linguistique et identitaire plongés dans un monde qui leur est totalement étranger, voulant ce qu'il y a de mieux pour leur enfant.

Tableau 1 Sentiments pouvant influencer le choix d'une langue signée par les parents d'enfant sourd

\begin{tabular}{|c|c|c|c|}
\hline & $\begin{array}{l}\text { Sentiment } \\
\text { d'incompétence }\end{array}$ & Sentiment d'incompréhension & Sentiments d'inquiétude \\
\hline $\begin{array}{l}\text { Sur le plan } \\
\text { linguistique }\end{array}$ & $\begin{array}{l}\text { Le parent est amené } \\
\text { à transmettre une } \\
\text { langue qu'il doit } \\
\text { apprendre }\end{array}$ & $\begin{array}{l}\text { Le parent est confronté à une } \\
\text { réalité linguistique qu'il croit } \\
\text { naturelle pour son enfant } \\
\text { (inversion de la transmission } \\
\text { linguistique) }\end{array}$ & $\begin{array}{l}\text { Le parent est inquiet vis-à- } \\
\text { vis de la "marginalité" d'une } \\
\text { socialisation passant par } \\
\text { une langue minoritaire }\end{array}$ \\
\hline $\begin{array}{l}\text { Sur le plan } \\
\text { identitaire }\end{array}$ & $\left|\begin{array}{llrr}\text { Le } & \text { parent } & \text { a } \\
\text { l'impression } & \text { de } & \text { ne } \\
\text { pas avoir } & \text { choisi } \\
\text { entre } & \text { l'oral } & \text { ou } & \text { le } \\
\text { gestuel } & & \end{array}\right|$ & $\begin{array}{l}\text { Le parent se demande comment } \\
\text { éduquer son enfant à l'aide } \\
\text { d'une langue qui l'amènera à } \\
\text { s'affilier avec une communauté } \\
\text { inaccessible au parent }\end{array}$ & $\begin{array}{l}\text { Le parent doit adopter une } \\
\text { trajectoire parentale qui } \\
\text { placerait l'enfant dans un } \\
\text { monde qui s'éteint } \\
\text { (augmentation }\end{array}$ \\
\hline
\end{tabular}




\section{Conclusion}

Cette recherche nous a permis de documenter l'expérience des parents en général. En nous concentrant sur les 52 entrevues effectuées au Canada avec des parents francophones d'enfant ayant une surdité, nous avons pu explorer les représentations de ces parents au sujet des langues signées et essayer de comprendre pourquoi si peu de familles font le choix d'une langue signée pour leur enfant atteint de surdité. Au départ, si les professionnels de la santé font une présentation complète des différentes options communicationnelles pour l'enfant sourd, cela donne la possibilité aux familles de prendre une décision éclairée que ce soit pour l'oralisme, les langues signées ou le bilinguisme. Par cette discussion avec les intervenants, cela donne le temps aux parents d'assimiler chaque option et de comprendre que chacune des décisions aura un impact sur le devenir de l'enfant ayant une surdité, mais également sur le reste des membres de la famille. Par la suite après avoir eu accès à ces informations, les parents vont faire un choix pour leur enfant qui va dépendre de leur identité, de leur éducation et de celle qu'ils veulent pour leur enfant.

Durant ces premiers mois après le diagnostic de surdité, les parents vont devoir devenir des experts dans le domaine de la surdité. Sachant que la majorité des parents d'enfant sourd n'ont jamais eu de contacts avec de personne sourde, les connaissances qu'ils peuvent avoir lorsqu'ils envisagent une langue des signes comme mode de communication pour leur enfant s'appuient sur des représentations portées par la société. Ce mode de pensée peut amener les parents à croire, comme nous l'avons vu à travers leur discours, que les langues signées viennent naturellement aux sourds, que ces langues ont tendance à isoler ces locuteurs ou encore que ces langues gestuelles sont vouées à disparaître.

Cette annonce de surdité de l'enfant peut faire émerger une panoplie de sentiments qui influence la disposition des parents à s'engager dans une communication orale plus que dans une démarche d'apprentissage et de transmission d'une langue signée avec leur enfant ayant une surdité. L'option des langues signées est-elle dévalorisée par certains professionnels lors de la présentation des différents modes de communication? Selon notre recherche, ces parents n'ont pas une connaissance entière du potentiel des langues signées, comme nous pouvons le voir dans le propos suivant :

Quand on nous dit surdité, on pense Sourds, langage des signes puis il n'y aucune solution puis en fait il y a plein de solutions, mais on ne nous le dit pas tout de suite (12-401-1).

Ces familles se retrouvent la plupart du temps sans référence pour imaginer qu'une langue des signes puisse devenir un vecteur de leur parentalité. L'accompagnement et le soutien des professionnels de la santé qui offrent des services publics et ceux de la communauté sourde peuvent aider à atténuer les sentiments d'incompétence, d'incompréhension et d'inquiétude des parents d'enfant sourd. Mais pour permettre à ces parents de pouvoir se penser, à travers une langue des signes, comme des agents de socialisation compétents, les parents ont besoin de s'identifier à celle-ci et de se l'approprier non pas seulement comme outil communicationnel, mais également comme façon de socialiser leur enfant sourd. 


\section{BIBLIOGRAPHIE}

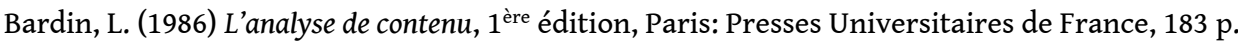
Boucher, N. (2001) Mise en jeu de la différence corporelle au Québec, Pour une sociologie du handicap. Thèse de doctorat, Université Laval, Québec.

Bouteyre, E., Jurion, M. \& C. Jourdan-Ionescu (2006) Remarques sur le vécu affectif de la fratrie de quelques enfants sourds, Neuropsychiatrie de l'enfance et de l'adolescence, 54, p. 117-124.

Calderon, R \& M. Greenberg (1999) Stress and coping in hearing mothers of children with hearing loss: factors affecting mother and child adjustment, American Annals of the Deaf, 144, 1, p. 7-18.

Charbonneau, J., \& P. Estèbe (2001) Entre l'engagement et l'obligation : l'appel à la responsabilité à l'ordre du jour, Lien social et Politiques, 46, p.5-15.

Clerebaut, N., (2005) Enfants entendants et parents sourds : aspects émotionnels et relationnels, Connaissances et surdité, 13, p.14-20.

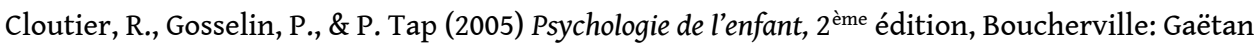
Morin Éditeur, 559 p.

Cuxac, C. (1983) Le langage des sourds, Paris: Payot, 206 p.

Dagenais, D. (2000) La fin de la famille moderne. Signification des transformations contemporaines de la famille, Québec: Les Presses de l'Université Laval, 267 p.

Dalle-Nazebi, S. (2014) Quand le bilinguisme entre dans la famille avec la naissance d'un enfant : Langue des signes et français au quotidien, Langage et société, 147, p. 23-34.

Delamotte-Legrand, S. \& R. Sabria (2001) L'enfant sourd et ses langues : l'unicité des choix en parcours langagiers pluriels, in Castelloti V., D'une langue à d'autres : pratiques et présentations, Rouen: Publication de l'Université de Rouen.

Delaporte, Y. (2002) Les sourds, c'est comme ça : Ethnologie de la surdimutité, Paris: Éditions de la Maison des sciences de l'homme, 398 p.

Deslaurier, J.-P. (1997) L'induction analytique, in Deslauriers, L.-H., Groulx, A., Laperrière, R., Mayer \& A. P. Pires (eds), La recherche qualitative, Québec: Gaëtan Morin, p. 293-307.

Dionne, C., Rousseau, N., Drouin, C., Vézina, C. \& S. McKinnon (2006) Expérience des familles dont un enfant présente une incapacité : perceptions et besoins actuels, Service social, 52, 1, p. 65-77.

Dubuisson, C \& R. Berthiaume (2005) Le rôle des parents dans une approche bilingue : la compétence des parents en LSQ et son impact sur la compétence de l'enfant, in Dubuisson, C. \& R. Berthiaume, Entre parents, parlons surdité, recueil de textes, Montréal: Groupe de recherche sur la LSQ et le bilinguisme sourd, UQAM.

Dubuisson, C. \& C. Grimard (2006) La surdité vue de près, Québec: Les Presses de l'Université du Québec, 428 p.

Flaherty, M. (2015) What We Can Learn from Hearing Parents of Deaf Children, Australasian Journal of Special Education, 39, 1, p.1-18.

Fortnum, H. M., Marshall, D. H. \& A. Q. Summerfield (2002) Epidemiology of the UK population of hearing-impaired children, including characteristics of those with and without cochlear implants-audiology, aetiology, comorbidity and affluence, Int J Audiol, 41, p.170-179. 
Fougeyrollas, P. (2010) La funambule, le fil et la toile: transformations réciproques du sens du handicap, Québec: Les Presses de l'Université Laval, 315 p.

Gardou, C. (1996) Parents d'enfant handicapé, le handicap en visage 2, Ramonville-Saint-Agne: Edition Ères, $185 \mathrm{p}$.

Gaucher, C. (2007) L'indiscutable différence des Sourds : intégration et pluralisme au sein des mondes occidentaux, in Vibert, S., Le pluralisme dans les sociétés modernes : culture, droit et politique, Montréal: Québec-Amérique.

Gaucher, C. (2009) Ma culture, c'est les mains. La quête identitaire des Sourds du Québec, Québec: Les Presses de l'Université Laval, 183 p.

Gaucher, C. (2012) Les Sourds ne gesticulent pas, « ils signent », Réflexion sur le rapport entre corps sourds et langues des signes, Anthropologie et Sociétés, 36, 3, p. 153-170.

Gaucher, C. \& L. Duchesne (2015) Votre enfant a une surdité ? Vous n'êtes pas seuls!, Québec: Fondation des Sourds du Québec, 123 p.

Giraud, C. (2011) Qu'est-ce que l'engagement ? Pairs: L'Harmattan, 287 p.

Hintermair, M. (2000) Hearing impairment, social network, and coping: the need for families with hearing-impaired children to relate to other parents and to hearing-impaired adults, American annals of the Deaf, 145, 1, p.41-53.

Jackson, C., Wegner, J-R. \& A-P. Turnbull (2010) Family quality of life following early identification of deafness, Language, Speech, and Hearing Services in Schools, 41, p. 194-205

Jackson, C., Wegner, J-R. \& A-P. Turnbull (2004) Impact of deafness on family life: A review of the literature, Topics in Early Childhood Special Education, 24, 1, p.15-27.

Lachance, N. (2002) Analyse du discours sur la culture sourde au Québec, Fondements historiques et réalité contemporaine, Thèse de doctorat du département d'anthropologie, Université de Montréal. Lachance, N. (2007) Territoire, transmission et culture sourde, Perspectives historiques et réalités contemporaines, Québec: Les Presses de l’Université Laval, 292 p.

Ladd, P. (1983) Intégration and génocide, 2LPE, 1, 2, p. 295-306.

Lagarrigue, J. (2011) Le jeune sourd et sa famille face à des points de vue divergents, Empan, 83, 3, p.74-78. doi:10.3917/empa.083.0074.

Lane, H. (1984) Quand l'esprit entend. Histoire des sourds-muets, Paris: Odile Jacob, 592 p.

Lane, H. (1993) Vue historique de la médicalisation de la surdité de culture, Psychanalyste : revue du Collège de psychanalystes, 46-47, p.173-187.

Lavigne, C. (2003) Handicap et parentalité, La surdité, le handicap mental et le pangolin. Paris: Éditions du CTNERHI, $357 \mathrm{p}$.

Lhéricel, B. (2006) Du choix de la langue pour l'enfant sourd, in Daigle, D. \& A.-M. Parisot, Surdité et société : perspectives psychosocial, didactique et linguistique, Québec: Presses de l'Université du Québec.

Luterman, D. (1987) Deafness in the Family, San Diego, CA: College Hill Press, 138 p.

Millet, A (2003) Les représentations sociales de la LSF : comment penser un sujet sourd bilingue et biculturel, La nouvelle revue de l'AIS, 23. p. 63-72 
Millet, A. (2006) Dynamique iconique en LSF aspects syntaxique et discursifs, in Daigle, D. \& A. M. Parisot, Surdité et société : Perspectives Psychosociale, Didactique et Linguistique, Québec: Presses Universitaires du Québec, p. 129-142

Millet, A. (1999) Orthographe et écriture, langage et surdité, système, représentations, variations, HDR Sciences du langage, Université Stendhal de Grenoble 3.

Minet, A. (2017) Une construction de la parentalité d'enfant handicapé par un engagement militant : Quand un imaginaire associatif devient fondateur d'une identité parentale, Paris: L'Harmattan, $276 \mathrm{p}$.

Mottez, B. (1977) À s'obstiner contre les déficiences, on augmente souvent le handicap : l'exemple des sourds, Sociologie et Sociétés, 9, 1, p. 20-32.

Mottez, B. (2006) Les Sourds existent-ils ? Textes réunis et présentés par Andrea Benvenuto, Paris: L'Harmattan, $388 \mathrm{p}$.

Nader-Grosbois, N. (2015) Psychologie du handicap, Paris: De Boeck Supérieur, 567 p.

Papousek H. \& M. Papousek (1987) Intuitive parenting: a dialectic counterpart to the infant's integrative competence, in Osofsky, J. D. (eds.), Handbook of infant development, New-York: Wiley \& Sons.

Perreault, S.-D. (1996) Les clercs de Saint-Viateur et l'Institution des sourds-muets, 1848-1930 : berceau de la communauté sourde Montréalaise, Mémoire de maîtrise, Ottawa.

Perreault, S. D. (2002) La naissance des communautés sourdes à Montréal entre 1880 et 1920, in Wallot, J.-P. Constructions identitaires et pratiques sociales, Ottawa: Presses de l'Université d'Ottawa, p. $147-162$.

Perreault, S.D. (2006) Une communauté qui fait signe : les Sœurs de Notre-Dame-des-Sept-Douleurs, 1887-2006, Paris: Carte Blanche.

Porter, A. \& S. Edirippulige (2007) Parents of deaf children seeking hearing loss related information on the internet: The Australian experience, Journal of deaf studies, 12, 4, p. 518-599.

Saillant, F. \& E. Gagnon (2001) De la dépendance et de l'accompagnement, Soins à domicile et liens sociaux. Québec: Les Presses Universitaires de Laval.

Scelles, R. (2002) Contribution de l'étude des situations de handicap à la compréhension de la vie psychique du sujet dans ses liens aux autres, Habilitation à diriger des thèses, Université de Rouen.

Stiker, H-J., 1997 [1982] (2005) Corps infirmes et Sociétés, Paris: Dunod. 248 p.

Therrien, C. \& J. Le Gall (2012) Nouvelles perspectives sur la mixité conjugale, Enfances, Familles, Générations, 17, p. 1-20.

Virole, B. (2006) Psychologie de la surdité, Questions de personne, Bruxelles: Broeck, $526 \mathrm{p}$.

Virole, B. (2011) La disparition des Sourds ?, Empan, 83, 3, p. 14-17.

Vrancken, D. \& C. Bartholomé (2004) L'accompagnement des personnes handicapées en Belgique : Un concept au cœur des nouvelles politiques sociales, Nouvelles pratiques sociales, 17, 1, p. 98-111. doi:10.7202/010576ar.

White, J. M. \& D. M. Klein (2008) Family Theories, Californie: Sage, 333 p.

Young, J., Robinson, M., Chell, S., Sanderson, D., Chaplin, S., Burns, E. \& J. Fear (2005) A prospective baseline study of frail older people before the introduction of an intermediate care service, Health \& Social Care in the Community, 13, p. 307-312. 


\section{NOTES}

1. Parentage intuitif: les comportements communicationnels (imitation des sons de l'enfant, ajustement du débit ou du niveau de langage, etc.) et performatifs (routine avec l'enfant, jeux, etc.) que les parents puisent à leur expérience biographique dans des registres implicites de leur propre éducation (Papousek \& Papousek, 1987).

2. Selon l'Organisation mondiale de la santé (2013), environ 32 millions d'enfants vivent avec une surdité dans le monde. L'étude britannique menée par Fortnum et al. en 2001 démontre que la prévalence de la surdité néonatale dans la population générale est de 1,12 par 1000 naissances vivantes. Tout porte à croire que cette prévalence vaut pour le Canada.

3. Ce sentiment de « manipulation » est ressorti dans le livre de Dubuisson \& Grimard (2006), par exemple, lorsque les auteures rapportent les propos d'une jeune fille sourde qui explique pourquoi ses parents ont choisi pour elle une communication orale : « Je comprends que ma mère ait subi l'influence des médecins qui font des pressions. C'est difficile, ils sont très crédibles » (p. 242).

4. Ce parent indique que la communication était plus ou moins bonne.

\section{RÉSUMÉS}

L'annonce de surdité de l'enfant peut être vécue comme un choc au sein de la famille. Dans la majorité des cas, les parents sont entendants et ont peu de connaissances sur la surdité. Pourtant, dès les premiers mois, ces parents vont devoir prendre une décision concernant le mode de communication de leur enfant. Le choix d'une communication en langue signée n'est que rarement envisagé par les parents. Cet article repose sur une recherche qualitative faite auprès de 52 parents d'enfant ayant une surdité au Canada et s'intéresse aux défis linguistiques et identitaires perçus par ces parents lorsque vient le temps d'inclure une langue signée au sein de l'environnement familial.

Receiving their child's hearing loss diagnosis can render parents in a state of shock, sending them suddenly to a world that is completely unknown (Jackson et al., 2004). In most cases, the parents are hearing persons and know very little about deafness; therefore, they feel lost in this new world (Clerebaut, 2005). During the first months, these parents are put into contact with a multitude of health professionals (audiologists, speech therapists, oto-rhino-laryngologists, etc). In addition, they will need to decide on a method of communication for their child. It is with the help of health, re-adaptation and education professionals that the parents will choose the path that they think is the most appropriate for their deaf child (Dubuisson \& Grimard, 2006). The families of deaf children are often torn, having to accept that their child is different whilst having to find a solution to the communication difficulties. A certain reflection period is therefore necessary in order for them to make the necessary and appropriate decisions with regards to the re-adaptation process for their child. The option of sign language communication is only rarely considered by parents as it goes against a collective norm related to the cultural process of language transmission (Dalle-Nazebi, 2014). Whether their decision tends towards oral communication or sign language, the child's deafness becomes the family's focal point and disrupts its stability (Jackson et al., 2010).

A study carried out with Canadian parents shows that the sign language option is often 
considered by these same parents as a source of awkwardness, that can be viewed as an obstacle to their commitment.

From a methodological point of view, this article will present a qualitative study of 52 parents of deaf children in Canada and look into the identity and language challenges perceived by these parents when it comes to incorporating a sign language within the family environment. Secondly, we will detail a few methodological points in order to set the context for the data and analysis described in this article. We will thereafter focus on how the discovery of deafness can impact the family life and parental commitment. Finally, we will see that the idea of including the sign language within the family environment can trigger, for the parents, different feelings with regards to language and identity transmission: incompetence, misunderstanding and concern.

INDEX

Mots-clés : parentalité, langue, surdité, communication, engagement

Keywords : parenthood, language, deafness, communication, commitment

\section{AUTEURS}

\section{SARAH KIRSCH}

École de travail social, Université de Moncton, Moncton, Canada

esk5566@umoncton.ca

\section{CHARLES GAUCHER}

École de travail social, Université de Moncton, Moncton, Canada charles.gaucher@umoncton.ca 